\title{
Effect of deposition rate on morphology evolution of metal-on-insulator films grown by pulsed laser deposition
}

\section{Citation}

Warrender, Jeffrey M. and Michael J. Aziz. 2007. Effect of deposition rate on morphology evolution of metal-on-insulator films grown by pulsed laser deposition. Phyical Review B 76: 045414 (8 pages).

\section{Published Version}

http://dx.doi.org/10.1103/PhysRevB.76.045414

\section{Permanent link}

http://nrs.harvard.edu/urn-3:HUL.InstRepos:2789367

\section{Terms of Use}

This article was downloaded from Harvard University's DASH repository, and is made available under the terms and conditions applicable to Other Posted Material, as set forth at http:// nrs.harvard.edu/urn-3:HUL.InstRepos:dash.current.terms-of-use\#LAA

\section{Share Your Story}

The Harvard community has made this article openly available.

Please share how this access benefits you. Submit a story.

\section{Accessibility}




\title{
Effect of Deposition Rate on Morphology Evolution of Metal-on-Insulator Films Grown by Pulsed Laser Deposition
}

Jeffrey M. Warrender ${ }^{1,2}$ \& Michael J. Aziz ${ }^{1}$

${ }^{1}$ Harvard School of Engineering and Applied Sciences, 29 Oxford St, Cambridge MA 02138

${ }^{2}$ U.S. Army Benét Laboratories, 1 Buffington St, Watervliet NY 12180.

Electronic Mail: jwarrend@post.harvard.edu

\begin{abstract}
Ag films were grown by pulsed laser deposition on insulating $\mathrm{SiO}_{2}$ and mica substrates and exhibited a morphological progression beginning with nucleation of $3 \mathrm{D}$ islands and culminating in a continuous, electrically conducting film. The rate of advancement through this progression with increasing pulse frequency was studied with experiments and with Kinetic Monte Carlo simulations. Experiments at $93^{\circ} \mathrm{C}$ and $135^{\circ} \mathrm{C}$ give exponents of -0.34 and -0.31 , respectively, for the scaling of the electrical percolation thickness with pulse frequency. Simulations predicted an exponent of -0.34 , in excellent agreement with the experiments. Both of these values agree well with the previously reported analytic value of -0.33 for the scaling of the morphology transition thickness with average flux in continuous deposition. Simulations also predicted that data collapse for island density vs. amount deposited would be observed for experiments run at the same value of the parameter $B / f$ at constant amount deposited per pulse, where $B$ is the kinetic rate constant for coalescence and $f$ is the pulse frequency. Measurements of the percolation transition were consistent with this prediction. These findings indicate that the elementary processes included in the KMC simulation — substrate terrace diffusion, irreversible aggregation of hemispherical islands, and two-island coalescence, but neglecting the effects of
\end{abstract}


kinetic energy - are sufficient to explain the behavior observed when the pulse rate is varied at constant kinetic energy.

PACS: 81.15.Fg; 68.55.-a; 81.07.Bc; 82.20.Wt 


\section{Introduction}

Pulsed laser deposition (PLD) is a thin film growth technique that differs from conventional deposition techniques, such as thermal evaporation, in at least two essential ways: the depositing species arrive in short bursts of 10-100 $\mu \mathrm{s}$; and the depositing species have kinetic energy $10-100 \mathrm{eV}$, compared with $<1 \mathrm{eV}$ for thermally-deposited films. PLD is useful as a research tool because it allows the instantaneous deposition flux and the average deposition flux to be varied independently and studied separately - a capability that is not possible in continuous flux delivery techniques. The instantaneous deposition flux is expected to govern the initial density of islands ${ }^{1}$, while the average deposition flux governs the growth rate of islands. Pulsed flux delivery is also of technological interest because it provides an additional kinetic handle on film growth that could permit improved control of film morphologies. ${ }^{2,3}$ In this work, we explore the influence of the average deposition flux during PLD growth at constant instantaneous flux, brought about by varying the laser pulse rate under otherwise identical laser, substrate, thermal, and background conditions. We chose $\mathrm{Ag}$ films on $\mathrm{SiO}_{2}$ as a model metal-oninsulator film growth system.

Metal-on-insulator thin films typically grow according to the Volmer-Weber growth mode, in which atoms grow in $3 \mathrm{D}$ islands on the surface. ${ }^{4-8}$ As the islands grow larger they impinge upon each other. Upon impingement, islands begin to coalesce, driven by capillary

forces toward a more equiaxed equilibrium shape, ${ }^{9}$ delaying the development of a contiguous film. Our use of the term "coalescence" encompasses the entire process by which two or more impinging equiaxed islands become a single, larger, equiaxed island. During this coalescence process, we refer to the multi-island system as a "coalescing cluster". This terminology is not 
universal in the literature. The characteristic time scale for the coalescence process increases as the participating islands grow larger, ${ }^{10}$ according to

$$
\tau=\frac{R^{4}}{B},
$$

where $R$ is the initial radius of the coalescing islands and $B$ is a temperature-dependent kinetic rate constant, which we refer to as the "coalescence parameter" with dimensions $\left[\right.$ length ${ }^{4}$ time $^{-1}$, given by

$$
B \equiv \frac{D_{s} S_{0} \gamma \Omega^{2}}{k_{B} T},
$$

with $D_{S}$ the surface self-diffusion coefficient of atoms on the island, $\gamma$ the surface energy, $\Omega$ the atomic volume, and $S_{0}$ the number of mobile surface atoms per unit area. ${ }^{6,10}$ Given a cluster consisting of a pair of coalescing islands, there will be an island size above which the characteristic time scale for coalescence exceeds the average time for a third island to grow to impinge with one of the pair. It is at this point that clusters of coalescing islands remain elongated on the surface; they have become kinetically frozen. ${ }^{6}$ Further deposition joins these elongated clusters, resulting in a tortuous network of bare channels that continues to fill in with subsequent deposition. ${ }^{5}$ Representative Atomic Force Microscope (AFM) images of the morphological progression are shown in Fig. 1.

There are at least two morphological transitions in this progression: an "elongation transition", after which the average object on the surface no longer has an equiaxed in-plane shape (coalescence has been "frozen out"); and a "percolation transition", after which the film conducts electrically over macroscopic distances. For the experiments that will be reported, the percolation transition is more readily monitored; for simulations, however, the elongation transition is more readily monitored. The thickness at which each transition occurs is expected 
to have the same scaling exponent with average flux ${ }^{11}$, and analytic models for continuous deposition predict that the transition thickness $d$ scales as $d \propto(B / F)^{1 / 3}$ where $F$ is the average

flux. ${ }^{6,11}$ In this work, $F$ is varied at constant instantaneous deposition flux by holding the pulse details (duration, amount deposited per pulse) constant and varying the delay between pulses; this is a capability not accessible in conventional continuous deposition experiments.

\section{Experimental Procedure}

Films were prepared by PLD in a vacuum chamber with base pressure of $2 \times 10^{-8}$ Torr. The target was $99.999 \%$ pure $\mathrm{Ag}$, and was irradiated with a $\mathrm{KrF}^{+}$excimer laser beam (Lambda Physik LPX 305), wavelength $248 \mathrm{~nm}$, pulse duration $30 \mathrm{~ns}$ FWHM. The laser spot size was $\sim 3 \mathrm{~mm} \times \sim 1 \mathrm{~mm}$, with an energy density of $\sim 5 \mathrm{~J} / \mathrm{cm}^{2}$. The target was rotated during deposition, and was irradiated for 2 minutes at $10 \mathrm{~Hz}$ to clean the target prior to each growth run. The target-to-substrate distance was $10.5 \mathrm{~cm}$. Films were grown on $\mathrm{SiO}_{2}$ and freshly cleaved muscovite mica.

The onset of the electrical percolation transition was monitored with an in situ resistance measurement. In order to make good electrical contact with the growing film, Ag contact pads, $300 \mathrm{~nm}$ thick, are deposited onto a clean $\mathrm{SiO}_{2}$ surface in a thermal evaporator. $\mathrm{SiO}_{2}$ was chosen as a substrate because previous studies have shown that $\mathrm{Ag}$ films on $\mathrm{SiO}_{2}$ behave in accordance with the predictions of classical nucleation theory. ${ }^{12}$ A shadow mask is used to produce two square pads of deposited silver $0.64 \mathrm{~cm}$ on a side, and $0.64 \mathrm{~mm}$ apart. Indium drops are applied to each of these pads, and Au-tipped contact probes are embedded in the In while it is molten. The In drops improve the electrical contact between the contact pads and the contact probes. Each probe is plugged into a Au-coated receptacle and is connected by a wire to an electrical 
feedthrough. The resistance measurement is performed by a Keithley 2000 digital multimeter, in the "4 Wire Resistance" mode. The resistance is recorded once per second. Based on the work of So et al., it is expected that when the sample becomes conducting, a precipitous drop in the resistance will be observed. ${ }^{13}$. As we described elsewhere, this is evidenced on a logarithmic graph of resistance $v s$. film thickness as a dramatic change in the slope that results in a drop in resistance of at least an order of magnitude. ${ }^{14}$ Films were analyzed with ex situ Atomic Force Microscopy (AFM) and Scanning Electron Microscopy (SEM) to check morphology.

The average deposition rate was measured before and after film growth by inserting a quartz crystal rate monitor into the flux. The deposition rate measurement was calibrated using Rutherford Backscattering Spectrometry. The deposition rate was approximately 0.006 $\mathrm{nm} /$ pulse.

\section{Experimental Results}

$\mathrm{Ag}$ films were grown on $\mathrm{SiO}_{2}$ by $\mathrm{PLD}$ at $40^{\circ} \mathrm{C}, 93^{\circ} \mathrm{C}$, and $135^{\circ} \mathrm{C}$ over a range of pulse frequencies. Plots of the thickness at which each film became electrically conducting vs. pulse rate are presented in Fig. 2. Errors in the reported percolation thickness (not shown) are estimated to be $8 \%$, having a $6 \%$ contribution from error associated with the deposition rate measurement and 5\% contribution associated with run-to-run variation in the average deposition rate. Panels (a) and (b) clearly show that a power law relationship accurately fits the "hightemperature" data, and that the scaling exponent is $-0.31 \pm 0.02$ for $135^{\circ} \mathrm{C}$ and $-0.339 \pm 0.002$ for $93^{\circ} \mathrm{C}$. Both are in excellent agreement with the scaling exponent of $-1 / 3$ obtained from analytical models for continuous deposition ${ }^{6,11}$ and with the value of -0.34 predicted by previous kinetic Monte Carlo simulations of pulsed deposition. ${ }^{15}$ In a recent paper, we observed that films in the 
equiaxed island stage grown by PLD at $110^{\circ} \mathrm{C}$ exhibited an island spacing of about $100 \mathrm{~nm}$ island center-to-island center. ${ }^{14}$

Panel (c) shows the behavior for much lower temperature, at $40^{\circ} \mathrm{C}$. A single power law cannot accurately describe these data. There appear to be several slopes present but the points demarking these transitions are difficult to identify precisely. A fit to the data for $f<10 \mathrm{~Hz}$ gave an exponent of -0.31 . A fit to the data for $2 \mathrm{~Hz}<f<20 \mathrm{~Hz}$ gave an exponent of -0.13 . Finally, the data show a high frequency regime, at $20 \mathrm{~Hz}$ and above, beyond which increasing the frequency further does not appear to change the percolation thickness further; the exponent in this region was -0.08 .

\section{Simulations}

Kinetic Monte Carlo (KMC) simulations were implemented to identify the impact of pulsed deposition on the Volmer-Weber morphological progression. The simulation algorithm is based on code described elsewhere. ${ }^{16}$ The simulation is performed on a square lattice, $512 \times$ 512. Atoms are added to the system by random deposition within a pulse, ignoring effects of kinetic energy. Atoms on the substrate may diffuse, with a diffusion constant given $D$ by $D=\frac{1}{4} a^{2} v \exp \left[-E_{D} / k_{B} T\right]$, with $a$ the minimum lattice translation distance, $v$ the vibration frequency, assumed equal to $1 \times 10^{13} / \mathrm{s}$, and $E_{D}$ the activation barrier for terrace diffusion. Atoms that impinge, either by deposition or diffusion, upon other atoms irreversibly nucleate a new island, and the system maintains a list that monitors each island's size and center of mass. Atoms that impinge upon an island are immediately and irreversibly relocated to the nearest vacant site to the projected center of mass of the island. This has the effect of forcing the islands 
to remain hemispherical, with a contact angle of $90^{\circ}$, which reproduces the Volmer-Weber film growth geometry. When two islands come into contact, the coalescence clock is started. After a time given by equation (1) has elapsed, with $R$ set equal to the radius of the smaller island, the pair is replaced with a new hemispherical island. The new island contains the same number of atoms as the sum of the atoms in the two-island system, and its center of mass is located at the center of mass of the original two-island system. ${ }^{11,15}$ An island may be involved in multiple coalescence processes simultaneously, which proceed independently except that the value of $R$ is updated after each coalescence event.

At each data-taking interval, the average number of constituent islands of a cluster, $N_{C}$, is obtained. When $N_{C}$ is significantly greater than unity, further deposition tends to cause other islands to impinge with a coalescing cluster in less time than will be required for the coalescence process to proceed significantly. $N_{C}=2$ is the point chosen to demark a transition from compact hemispherical islands to elongated clusters. The laterally averaged film thickness at which $N_{C}=$ 2 is defined as the elongation transition thickness $d_{\text {Elong. }}{ }^{11}$ To obtain better statistics, the plot of $N_{C} v s$. pulse number during a deposition simulation is fit with an exponential fit,

$$
N_{C}=1+A e^{p / p_{0}}
$$

for which $p$ is the pulse number, $A$ and $p_{0}$ are fitting parameters. Equation (3) was found to fit the curves of $N_{C} v s . p$ quite well. The quantity of interest is $p_{\text {Elong }}$ the pulse number at which $N_{C}$ $=2$, which is given by

$$
p_{\text {Elong }}=p_{0} \ln \left(A^{-1}\right) \text {. }
$$

The pulse duration was fixed for all pulsing rates $f$, at $100 \mu$ s, chosen based on the typical lifetime of the PLD plume (on the order of 10-100 $\mu$ s). Similarly, the amount deposited during the pulse, $I$, was held constant for all $f$, and the instantaneous deposition rate during the "on" 
portion of the pulse, $F_{\text {inst }}$, was also held constant. The substrate diffusion constant is varied by varying the ratio $E_{\mathrm{D}} / k_{B} T$.

Note that because of computational limitations, our simulations were performed to thicknesses of only several monolayers (ML), whereas the experiments show percolation thicknesses on the order of tens of $\mathrm{nm}$. Even considering that the elongation transition must occur at a lower thickness than the percolation transition, there is still a considerable difference in the sizes of islands studied by the simulations and experiments. The length scale of the film growth, and the resulting thickness at percolation, is controlled primarily by three parameters: the coalescence parameter $B$, the average deposition flux, and the ratio of the instantaneous deposition rate to the substrate surface diffusion constant (the $D / F$ ratio). Changes to either of the first two will affect the intercept of the percolation thickness $v s$. average deposition flux curve, but not its slope. Changing the $D / F$ ratio can, as shown below, cause growth to be in one of several different growth regimes, but does not change the exponent within a given regime. Therefore, even though the length scales of the simulations and experiments are different, comparison of the scaling exponents that emerge from each is warranted.

\section{Simulation Results}

The rate at which the islands increase in size is influenced primarily by the average deposition flux, whereas the rate at which the islands actually impinge is influenced also by the typical island size, separation, and time of nucleation, parameters that are dependent on the instantaneous deposition flux. Jensen treated the interrelationships between the pulse duration $t_{\text {pulse }}$, the pulse frequency $f$, and the characteristic monomer lifetime $\tau_{D}$, as giving rise to three different regimes: a "fast diffusion" regime, in which $t_{\text {pulse }} \gg \tau_{D}$, an intermediate regime, in which $t_{\text {pulse }}<\tau_{D}<1 / f$, and a very slow diffusion regime in which $\tau_{D} \gg 1 / f$. Jensen reported 
that the first and last of these effectively resembled continuous deposition; the first, because all surface processes occur when the pulse in on, the latter because surface processes occur over many pulses. ${ }^{3}$

The simulations demonstrate the complexity of the interaction between these effects. In Fig. 3 (a)-(c) we show simulation results for three different values of the substrate diffusion constant $D$. These panels plot the film thickness at the elongation transition vs. the pulse frequency. Panel (a) shows simulation results for $D=1.6 \times 10^{5} a^{2} / \mathrm{s}$, with $I=0.025 \mathrm{ML} / \mathrm{pulse}$, $B=500 a^{4} / \mathrm{s}$, where $a$ is the minimum lattice translation distance. This corresponds to fast diffusion, with $\tau_{D}$ comparable to $t_{\text {puse }}$. A single power law describes the transition thickness $v s$. frequency behavior for the range of frequencies examined here. Because diffusion is fast, the monomer population decreases after the pulse shuts off, and does so very rapidly relative to the time scale of the flux pulsing and of coalescence. In this limit, the growth can be thought of as occurring in bursts, followed by periods of variable duration in which coalescence is the only active surface process (because there are no monomers left to diffuse). The elongation thickness $v s$. pulse frequency exhibits a scaling exponent of $-0.345 \pm 0.002$. This is quite close to the scaling exponent of the experimentally measured percolation thickness of -0.31 and -0.34 reported above for $135^{\circ} \mathrm{C}$ and $93^{\circ} \mathrm{C}$. It also is close to the value -0.29 obtained from the simulation work of Carrey and Maurice, and to the exponent of -0.33 obtained from their analytical work and the work of Jeffers et al. ${ }^{6,11}$ This suggests that for the fast-diffusion regime, pulsed deposition behaves similarly to continuous deposition, with the average deposition flux being proportional to the pulse rate.

In panel (b) we show results for $D=1.7 \times 10^{3} a^{2} / \mathrm{s}, I=0.025 \mathrm{ML} /$ pulse, $B=500 a^{4} / \mathrm{s}$, corresponding to "intermediate" diffusion, with $t_{\text {pulse }}<\tau_{D}<1 / f$. An elbow in the data reveals 
subtleties not present for the case presented in panel (a). A low frequency regime, $f<30 \mathrm{~Hz}$, exhibits a scaling exponent of $-0.29 \pm 0.009$; for $f>30 \mathrm{~Hz}$ the scaling exponent is $-0.44 \pm 0.017$. A plot of island density for a single pulse $v s$. time ${ }^{17}$ for this value of $D$ shows that the island density saturates at about $0.05-0.1 \mathrm{~s}$. For pulse frequencies below $30 \mathrm{~Hz}$, then, there is no difference in nucleation or growth behavior, and so the only difference between frequencies is the amount of time allowed for coalescence. This frequency range is similar to the fast diffusion case of (a), with the difference that for the "fast" diffusion case, appreciable island nucleation and growth is observed while deposition is still active, whereas for "intermediate" diffusion, almost all nucleation occurs after the pulse ceases. For frequencies greater than $30 \mathrm{~Hz}$, monomers persist for the entire period between pulses, and the enhanced monomer population at the arrival of the subsequent pulse results in more nucleation events. The higher total island density results in an increased rate of impingement, and as a result, an earlier onset of the elongation transition. Of course, superposed on this effect is the reduction of time allowed for coalescence associated with decreasing time between pulses as the pulse frequency is increased. This presence of an additional condition that hastens the onset of elongation with increasing frequency can adequately explain why the exponent for $f>30 \mathrm{~Hz}$ is steeper than for $f<30 \mathrm{~Hz}$.

In Fig. 3(c) we show results for $D=1.0 \times 10^{-2} a^{2} / \mathrm{s}, I=0.01 \mathrm{ML} /$ pulse, for two different values of $B: 10 a^{4} / \mathrm{s}$ (diamonds) and $1 a^{4} / \mathrm{s}$ (crosses). The low value of $D$ represents a case of essentially no diffusion. If, additionally, coalescence is infinitely fast relative to deposition, this is the regime of droplet growth, which has been treated elsewhere. ${ }^{18,19}$ When coalescence occurs at a finite rate, differences upon varying the average deposition rate can be observed. The low values of $B$ used in the simulations shown in panel (c) represent very slow coalescence. At the lowest frequencies, some coalescence can occur, and so varying the pulse frequency again 
corresponds to varying the average deposition rate; the competition between coalescence and average deposition rate gives an exponent of $-0.34 \pm 0.05\left(B=1 a^{4} / \mathrm{s}\right)$ and $-0.35 \pm 0.01\left(B=10 a^{4} / \mathrm{s}\right)$ for elongation thickness $v$ s. pulse rate, just as was seen in Fig. 3(a), and consistent with the experimental exponents from Fig. 2(a) and (b).

The most remarkable result of panel (c), however, is the high-frequency regime, in which the exponent is -0.03 . Much insight can be gained by considering the time-dependent quantity

$$
\zeta \equiv \tau_{\text {coal }} /(1 / f)
$$

where $\tau_{\text {coal }}$, calculated using Eq. (1), is the time required for coalescence of a system consisting of two islands of the average coalescing size (obtained by finding the average size of all islands that begin coalescing during the time step in question) and $f$ is the pulse frequency. $\zeta$ is the ratio of the time required for coalescence to the time allowed for coalescence between pulses; in other words, it is the number of inter-pulse periods that a typical new coalescence event would require to complete. The value of $\zeta$ was obtained at the pulse number $p$ at which $N_{C}=2$, and this value is denoted $\zeta_{\text {elong }}$ and plotted in Fig. 3(d) for all three cases discussed above.

For the lowest temperature, at high frequency, the values of $\zeta_{\text {elong }}$ are very large; for example, for $B=10 a^{4} / \mathrm{s}$, at $800 \mathrm{~Hz}$, even from the beginning of the simulation, the time required for coalescence is over 200 times the delay between pulses. For these high frequencies, then, no appreciable amount of coalescence occurs during the simulation, and the difference between such frequencies is negligible with respect to coalescence. This explains the flat slope at high frequency in Fig. 3(c).

The results shown in panel (d) also corroborate the location of the elbows observed in panels (a)-(c). The highest temperature series in Fig. 3(d) shows no elbow, just as in panel (a). The elbows for the lowest temperature series occur at 10 and $100 \mathrm{~Hz}$ for $B=1$ and $10 \mathrm{a}^{4} / \mathrm{s}$, 
respectively, and the elbow at intermediate temperature occurs around 50-200 $\mathrm{Hz}$, near but slightly above the elbow in panel (b). Nevertheless, the elbow in the intermediate-temperature data and those in the low-temperature data in panel (d) are opposite in sense, as are the elbows in panels (b) and (c).

The low-frequency values of $\zeta_{\text {elong }}$ are roughly the same for the greatly varied input conditions of panels (a)-(c), and they exhibit at most a very weak dependence on pulse frequency. This suggests an independent method to experimentally determine the quantity $B$ in future work. By using equations (1) and (5), varying $f$ and measuring the average island radius $R$ at elongation, a fit for $B$ could be obtained.

The high frequency regime at low temperature, Fig. 3(c), is consistent with the highfrequency regime observed in the experimental results shown in Fig. 2(c). (The other regimes of Fig. 2(c) may be associated with different regimes of nucleation behavior as discussed in the interpretation of Fig. 3(b), but there is not an exact correspondence with the simulations). It is possible to give an estimate of the coalescence parameter $B$ that would result in the positioning of the main elbow observed at $20 \mathrm{~Hz}$ in Fig. 2(c). From Fig. 3(c), the onset of the "high frequency" regime, for slow coalescence, occurs at a value of $B / f \approx 0.1 a^{4}$. With $a=2.89 \times 10^{-8}$ $\mathrm{cm}$ for $\mathrm{Ag}$, the onset of the high frequency regime in the experimental data, Fig. 2(c), at $20 \mathrm{~Hz}$, would correspond to $B=1.40 \times 10^{-30} \mathrm{~cm}^{4} / \mathrm{s}$ at $40^{\circ} \mathrm{C}$. Bréchignac et al. reported experimental observations of fragmentation of fractal islands composed of Ag nanoclusters on graphite. They found that features having a branch width of $15 \mathrm{~nm}$ were observed to fragment in 2000s, while features having a branch width of $25 \mathrm{~nm}$ did not fragment. ${ }^{20}$ These observations provide estimates of a lower and upper limit for $B$, respectively. Using equation (1), with $15 \mathrm{~nm}$ and 25 $\mathrm{nm}$ supplied for $2 R, B$ should be between $1.58 \times 10^{-28} \mathrm{~cm}^{4} / \mathrm{s}$ and $1.22 \times 10^{-27} \mathrm{~cm}^{4} / \mathrm{s}$. These values 
are more than two orders of magnitude greater than our calculated value for $B$. We consider two possible explanations. The first possibility is that Ag monomer diffusion on graphite, which is not included in our interpretation, is contributing significantly to transport in the experiments of Bréchignac et al. A second possibility is that the kinetic rules used in the simulations, particularly the assumption that equations (1)-(2) accurately describe coalescence, could be invalid for our experimental conditions at $40^{\circ} \mathrm{C} .^{21}$ Equations (1)-(2) are strictly valid only at temperatures above the thermodynamic roughening temperature of the island surface: the presence of facets on the islands have been shown by KMC simulations ${ }^{22,23}$ and MD simulations ${ }^{24,25}$ to alter the kinetics of coalescence. For example, Combe and Jensen predicted that the $R^{4}$ dependence of equation (1) should be valid only above $800 \mathrm{~K}$. At lower temperatures, their simulations yield the prediction that equation (1) should be replaced by

$$
\tau=\frac{R^{\alpha}}{B}
$$

with an increasingly larger power of $\alpha$ observed as the temperature is decreased; they predict a power of 16.2 for an elongated island at $400 \mathrm{~K}$, which is a dramatic departure from equation (1). ${ }^{22}$

To test the implications of this prediction for the interpretation of our experimental results, we performed additional KMC simulations under the same conditions as those of Fig. 3(c) (diamonds), but for which the coalescence time was given by equation (6). $\alpha$ was set equal to 6 and 10, instead of the $\alpha=4$ dependence used in the other simulations. However, evaluating $B$ from the elbows in these simulations and from the observations of Bréchignac et al. using the same values of $\alpha>4$ only increases the discrepancy reported in the previous paragraph. Our 
inability to unify the results at $40{ }^{\circ} \mathrm{C}$ with the others appears to be a consequence of insufficiently well understood kinetics of morphology evolution of faceted nanocrystals.

We performed additional simulations with $\alpha$ equal to 5,6 , and 10 , with all other parameters the same as in Fig. 3(a). The simulations resulted in an elongation thickness vs. frequency behavior best fit by an exponent of $-0.345 \pm 0.002$ for $\alpha=4,-0.26 \pm 0.01$ for $\alpha=5$, $-0.223 \pm 0.006$ for $\alpha=6$, and $-0.13 \pm 0.01$ for $\alpha=10$. Our experimental observation for PLD at $135^{\circ} \mathrm{C}$ and $93^{\circ} \mathrm{C}$ of an exponent of $-0.31 \pm 0.02$ and $-0.339 \pm 0.002$, respectively, is consistent with the power $\alpha=4$ in equation (1) and is inconsistent with the predicted exponents for higher values of $\alpha$. This indicates that the kinetics of equations (1)-(2) appear to continue to describe PLD at temperatures as low as $93^{\circ} \mathrm{C}$. Kinetic roughening in PLD may play a role in extending the validity of the classical power law below the thermodynamic roughening transition temperature, or a mechanism resulting from the high kinetic energy of the depositing species could be influencing the film growth. We have recently reported the influence of kinetic energy effects on morphological evolution, which was evidenced by accelerated advancement through the characteristic progression as kinetic energy of the depositing species is systematically increased above $28 \mathrm{eV}$. We found that a transition in the vertical aspect ratio of the islands in the presence of sufficient impact-induced adatom-vacancy pair creation could explain this observation. ${ }^{14}$

\section{Data collapse for constant values of $B / f$}

A curiosity of Fig. 3(c) is the extent to which the $B=1 a^{4} / \mathrm{s}$ curve appears to be exactly the same as the $B=10 a^{4} / \mathrm{s}$, except shifted to the left; the elbow of the former occurs at $10 \mathrm{~Hz}$, and of the latter, at $100 \mathrm{~Hz}$. The ratio of these frequencies, 10, is the same as the ratio of the constants $B$. This suggests that a data collapse may be possible. 
First, we show that simulations run with the same value for the ratio $B / f$ give data collapse. Two different series were performed; one, under "fast diffusion" conditions, $D=1.6 \times 10^{5} a^{2} / \mathrm{s}$, with $I=0.025 \mathrm{ML} /$ pulse, $B / f=5 a^{4}$; the other, under "no diffusion" conditions, with $D=1.0 \times 10^{-2} a^{2} / \mathrm{s}$ and $I=0.01 \mathrm{ML} / \mathrm{pulse}, B / f=10 a^{4}$. The results of these simulations are shown in Fig. 4 (a) and (b), with the total ("large") island count plotted against pulse number. Fig. 4 plots raw data; no transformation was required. In both cases, the data collapse is excellent, suggesting that $B / f$ is indeed a dimensionless parameter of this system at constant amount deposited per pulse $I$.

This is a potentially difficult prediction to confirm experimentally, because both $B$ and the substrate surface diffusion constant $D$ are temperature-dependent. However, $D$ influences the initial island density more than island growth and impingement, and as we reported elsewhere ${ }^{17}$, the effect of island density (by way of instantaneous deposition rate, in that work) on transition thickness scaling is weak, and is dominated by the average flux/coalescence competition that results in the onset of the elongation transition. Thus, we assume that, as temperature is varied over a limited range, the influence of the variation of $D$ on percolation thickness will be weak and indirect while the influence of $B$ will be substantial and direct.

With this assumption, we seek to compare the morphology evolution for two films grown at equal values of $B / f$, at constant amount deposited per pulse. Of course, a detailed experimental comparison of the total island count $v s$. pulse number for each frequency and temperature was impractical, but comparing the percolation thickness should give a good estimate of the quality of the data collapse. Our approach is to compare the percolation thickness of films grown at the same ratio $B / f$. Practically speaking, this entails examining films grown at two different 
temperatures, $T_{1}$ and $T_{2}$, and pulse frequencies, $f_{1}$ and $f_{2}$, such that the ratio of the pulse frequencies $\rho$ is the same as the ratio of the coalescence parameters, or

$$
\rho=\frac{B_{1}}{B_{2}}=\frac{f_{1}}{f_{2}} .
$$

We examined our percolation thickness vs. frequency data at $135^{\circ} \mathrm{C}$ and $93^{\circ} \mathrm{C}$ (the data from Fig. 2(a) and (b)), and obtained the ratio of pulse frequencies that would enable the two plots to collapse onto each other. This is achieved by scaling the frequency axis of the $93^{\circ} \mathrm{C}$ data by $\rho$, performing a least-squares fit of the $135^{\circ} \mathrm{C}$ data together with the frequency-scaled $93^{\circ} \mathrm{C}$ data at varying $\rho$, and determining what $\rho$ gives the highest $R^{2}$ value in the least-squares fit. The result we obtained for these series is $\rho=2.29$. This means that the ratio of the coalescence parameters at these temperatures should also be 2.29 . If this is true, then $B_{1} / f_{1}=B_{2} / f_{2}$, and since we already know the data collapses when $f_{1}=\rho f_{2}$, we can claim that the KMC prediction has been corroborated.

For two temperatures $T_{1}$ and $T_{2}$, the ratio of the respective coalescence parameters $B_{1}$ and $B_{2}$ is, using (2)

$$
\rho=\frac{B_{1}}{B_{2}}=\frac{T_{2}}{T_{1}} \exp \left(-\frac{\varepsilon}{k_{B}}\left[\frac{1}{T_{1}}-\frac{1}{T_{2}}\right]\right)
$$

where $\varepsilon$ is the sum of the activation energies for formation and migration of $\mathrm{Ag}$ atoms on $\mathrm{Ag}$ islands. Solving for $\varepsilon$ for $\rho=2.29$ gives $\varepsilon=0.287 \mathrm{eV}$. Pomeroy reported MD-calculated barriers of $0.22 \mathrm{eV}$ and $0.3 \mathrm{eV}$ for step edge diffusion on $\mathrm{Ag}(111)$ along A- and B-steps, respectively, and these are consistent with calculations by Nelson et al. and Schlößer et al. ${ }^{26-28}$ If coalescence were instead mediated by terrace diffusion, the formation of $\mathrm{Ag}$ terrace adatoms from Ag steps would be rate-limiting. Pomeroy found the barrier for formation of a terrace 
adatom (having no in-plane nearest neighbors) from a kink site (having 3 in-plane nearest neighbors) to be $0.748 \mathrm{eV}$ in $\mathrm{Cu}(111)$, and given the similarity of the barriers for surface processes for $\mathrm{Ag}$ (111) and $\mathrm{Cu}(111)$, it would be quite surprising for the same process to have a barrier much lower than this for $\mathrm{Ag}(111){ }^{26}$ We therefore consider $\rho=2.29$ to be a credible value for the ratio $B_{1} / B_{2}$ for $T_{1}=135^{\circ} \mathrm{C}$ and $T_{2}=93^{\circ} \mathrm{C}$, and to indicate that coalescence is likely mediated by step edge diffusion rather than terrace diffusion over this temperature range. The experiments support the prediction that data collapse is observed for a constant value of the ratio $B / f$ for constant pulse details.

\section{Conclusion}

The effects of varying the average deposition rate by varying the laser pulse rate were discussed. Experiments at $93^{\circ} \mathrm{C}$ and $135^{\circ} \mathrm{C}$ gave exponents of -0.34 and -0.31 , respectively, for the scaling of percolation thickness with pulse frequency. Kinetic Monte Carlo simulations capable of modeling growth of hemispherical islands with island-island coalescence gave an exponent of -0.34 for the high-temperature, "fast diffusion" regime and for the low frequency range in the low-temperature "no diffusion" regime. The exponents from simulation and experiment agree well with each other, and with previously reported theoretical predictions. This agreement indicates that the kinetics described by equations (1) and (2) are valid for PLD deposition at temperatures as low as $93^{\circ} \mathrm{C}$, which is a surprising result given previously reported predictions that equation (1) should not accurately describe the kinetics of cluster coalescence below the roughening transition. For the case of slow diffusion and slow coalescence, the simulations predict a high frequency "freezing out" of coalescence leading to a "flat" region in the percolation thickness $v s$. pulse frequency curve. Experiments at $40^{\circ} \mathrm{C}$ were qualitatively 
consistent with this result. Simulations also predict that data collapse for island density vs. amount deposited can be achieved when the parameter $B / f$ is maintained. Experimental results demonstrated that films grown at the same value of $B / f$ achieve percolation at the same film thickness, which is consistent with the prediction.

The following picture emerges from our work. Kinetic energy effects are essential in determining the differences in morphological evolution when kinetic energy is systematically varied (such as in PLD vs. thermal deposition). As demonstrated by our previously reported work, increasing kinetic energy increases the vertical aspect ratio of the islands, resulting in a delay in the advancement through the characteristic morphological progression. However, the changes observed in the present work as the PLD pulse rate is varied at constant kinetic energy (and consequently, at constant vertical aspect ratio) are described adequately by the elementary processes included in the non-energetic KMC simulation - substrate terrace diffusion, irreversible aggregation of hemispherical islands, and two-island coalescence.

This research was supported by NSF Grant DMR-0306997. We gratefully acknowledge helpful discussions with Julian Carrey, Craig Arnold, and Jonah Erlebacher.

\section{References}

${ }^{1}$ D. Walton, J. Chem. Phys. 37, 2182 (1962).

${ }^{2}$ M.A. Gallivan, D.G. Goodwin, and R.M. Murray, Physical Review B 70, (2004).

${ }^{3}$ P. Jensen and B. Niemeyer, Surf. Sci. 384, L823 (1997).

${ }^{4}$ D.W. Pashley, M.H. Jacobs, M.J. Stowell, and T.J. Law, Philosophical Magazine 10, 127 (1964).

${ }^{5}$ A.A. Baski and H. Fuchs, Surface Science 313, 275 (1994). 
${ }^{6}$ G. Jeffers, M.A. Dubson, and P.M. Duxbury, Journal of Applied Physics 75, 5016 (1994).

${ }^{7}$ X. Yu, P.M. Duxbury, G. Jeffers, and M.A. Dubson, Physical Review B (Condensed Matter) 44, $13163(1991)$.

${ }^{8}$ P. Brault, A.-L. Thomann, and C. Andreazza-Vignolle, Surface Science 406, L597 (1998).

${ }^{9}$ Grain boundaries do not affect this picture very significantly when the boundary energy is much less than the metal free surface energy. The dihedral angles of grain boundary grooves in Ag vary from $160^{\circ}$ to $175^{\circ}$, according to A.P. Greenough and R. King, "Grain-Boundary Energies in Silver", J. Inst. Metals 79, 415 (1951).

${ }^{10}$ F.A. Nichols and W.W. Mullins, Journal of Applied Physics 36, 1826 (1965).

${ }^{11}$ J. Carrey and J.-L. Maurice, Physical Review B (Condensed Matter and Materials Physics) 63, 245408/1 (2001).

${ }^{12}$ A.A. Schmidt, H. Eggers, K. Herwig, and R. Anton, Surface Science 349, 301 (1996).

${ }^{13}$ S.K. So, H.H. Fong, C.F. Yeung, and N.H. Cheung, Applied Physics Letters 77, 1099 (2000).

${ }^{14}$ J.M. Warrender and M.J. Aziz, Phys. Rev. B 75, 085433 (2007).

${ }^{15}$ J.M. Warrender and M.J. Aziz, Applied Physics A 79, 713 (2004).

${ }^{16}$ J.D. Erlebacher, "Kinetic Rate Law Issues in the Morphological Relaxation of Rippled Crystal Surfaces," in Dynamics of Crystal Surfaces and Interfaces, edited by P.M. Duxbury and T. Pence (Plenum, New York, 1997).

${ }^{17}$ J.M. Warrender, "Morphological Evolution of Nanocrystal Metal-on-Insulator Films Grown by Pulsed Laser Deposition", Ph.D. Thesis, Harvard University, 2004.

${ }^{18}$ J. Carrey and J.-L. Maurice, Physical Review B (Condensed Matter and Materials Physics) 65, 205401/1 (2002).

${ }^{19}$ P. Meakin, Reports on Progress in Physics 55, 157 (1992). 
${ }^{20}$ C. Brechignac, P. Cahuzac, F. Carlier, C. Colliex, J. Leroux, A. Masson, B. Yoon, and U. Landman, Physical Review Letters 88, 196103 (2002).

${ }^{21}$ The position of the elbow could depend on the deposition flux; a higher flux could "freeze out" coalescence at a lower frequency, which would increase the value of $B / f$ used in our calculation above. In our experiments, we deposited approximately 0.025 ML per pulse, which is about twice the amount deposited per pulse in the simulations. The lower deposition rate in the simulations was essential to be able to get adequate statistics with which to fit the data with Eq. 3. We see no way for a factor of two in deposition flux to resolve a two order-of-magnitude discrepancy in the inferred value of $B$.

${ }^{22}$ N. Combe, P. Jensen, and A. Pimpinelli, Physical Review Letters 85, 110 (2000).

${ }^{23}$ P. Jensen and N. Combe, Computational Materials Science 24, 78 (2002).

${ }^{24}$ X. Yu and P.M. Duxbury, Physical Review B (Condensed Matter) 52, 2102 (1995).

${ }^{25}$ H.B. Liu, M. Jose-Yacaman, R. Perez, and J.A. Ascencio, Appl. Phys. A 77, 63 (2003).

${ }^{26}$ J. Pomeroy, "Hyperthermal Ion Epitaxy of Thin Metal Films", Ph.D. Thesis, Cornell University, 2002.

${ }^{27}$ R.C. Nelson, T.L. Einstein, and S.V. Khare, Surface Science 295, 462 (1993).

${ }^{28}$ D.C. Schlößer, K. Morgenstern, L. Verheij, G. Rosenfeld, F. Besenbacher, and G. Comsa, Surface Science 465, 19 (2000).

\section{Figure Captions}

Fig. 1. AFM images of morphological progression of PLD deposited Ag on mica. Scale bars are $1 \mu \mathrm{m}$. Principal stages are (a) island growth and coalescence (b) percolation and (c) hole filling. 
Fig. 2. (color online) Experimental film thickness at electrical percolation transition vs. pulse frequency for (a) $135^{\circ} \mathrm{C}$, (b) $93^{\circ} \mathrm{C}$, and (c) $40^{\circ} \mathrm{C}$. The data are well fit by a power law with a single exponent of about -0.33 for (a) and (b). Several power-law fits to the data are provided in (c) as a guide to the eye. The uncertainty in measured percolation thickness is $+/-8 \%$ for all points.

Fig. 3. (color online) KMC simulations of elongation transition. (a)-(c) Film thickness at elongation transition vs. pulse frequency for three different ratios of substrate surface diffusion constant $D$ : (a) $D=1.6 \times 10^{5} a^{2} / \mathrm{s}$ (fast diffusion), (b) $D=1.7 \times 10^{3} a^{2} / \mathrm{s}$ (slow diffusion) and (c) $D=1.0 \times 10^{-2} \mathrm{a}^{2} / \mathrm{s}$ (essentially no diffusion). Other parameters: (a) and (b) $\mathrm{I}=0.025 \mathrm{ML} / \mathrm{pulse}$, $B=500 \mathrm{a}^{4} / \mathrm{s}$. (c) $\mathrm{I}=0.01 \mathrm{ML} /$ pulse, and $B=10 \mathrm{a}^{4} / \mathrm{s}$ (diamonds) and $B=1 \mathrm{a}^{4} / \mathrm{s}$ (crosses). (d) Value of $\zeta$ (Eq. (5)) at the moment of the elongation transition, for the conditions of (a)-(c). Panels (a) and (b) adapted from Ref. 15.

Fig. 4. (color online) Data collapse for equal values of $B / f$. (a) $B / f=10 \mathrm{a}^{4}, D=1.6 \times 10^{5} \mathrm{a}^{2} / \mathrm{s}$. (b) $B / f=5 \mathrm{a}^{4}, D=1.0 \times 10^{-2} \mathrm{a}^{2} / \mathrm{s}$. Scatter at low pulse number is noise associated with the operation of the island counting algorithm in the absence of a clear minimum below which "small" sizes could be neglected. 


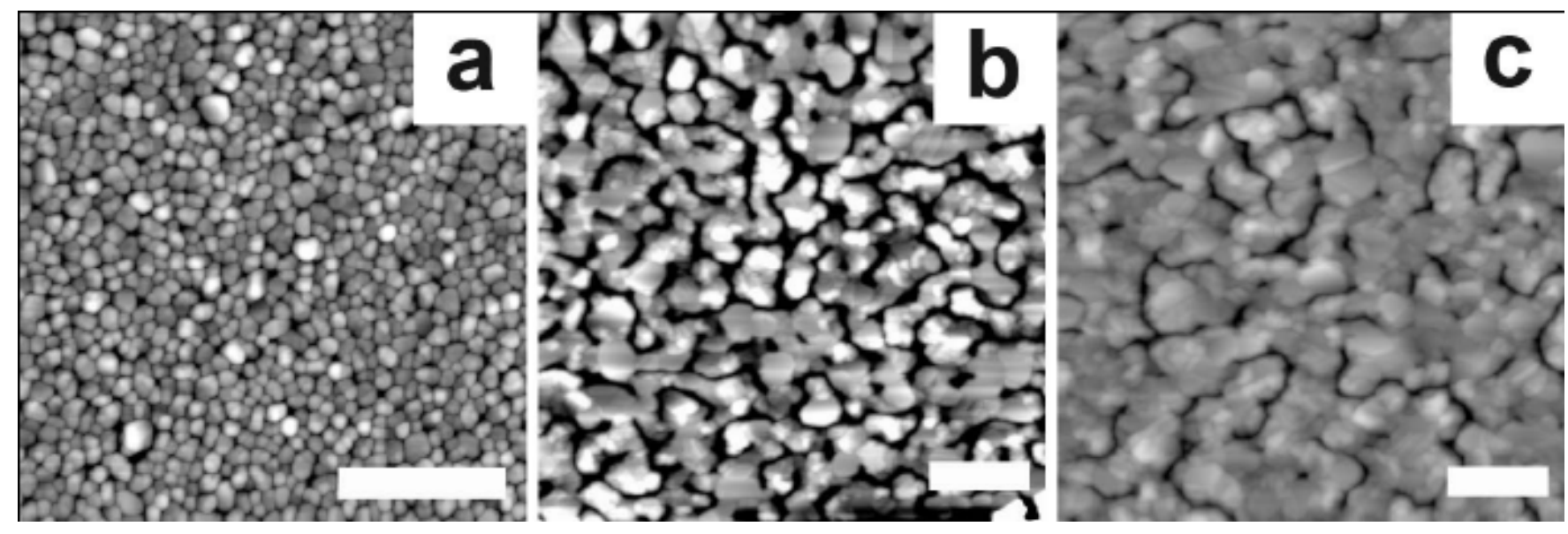

Fig. 1 


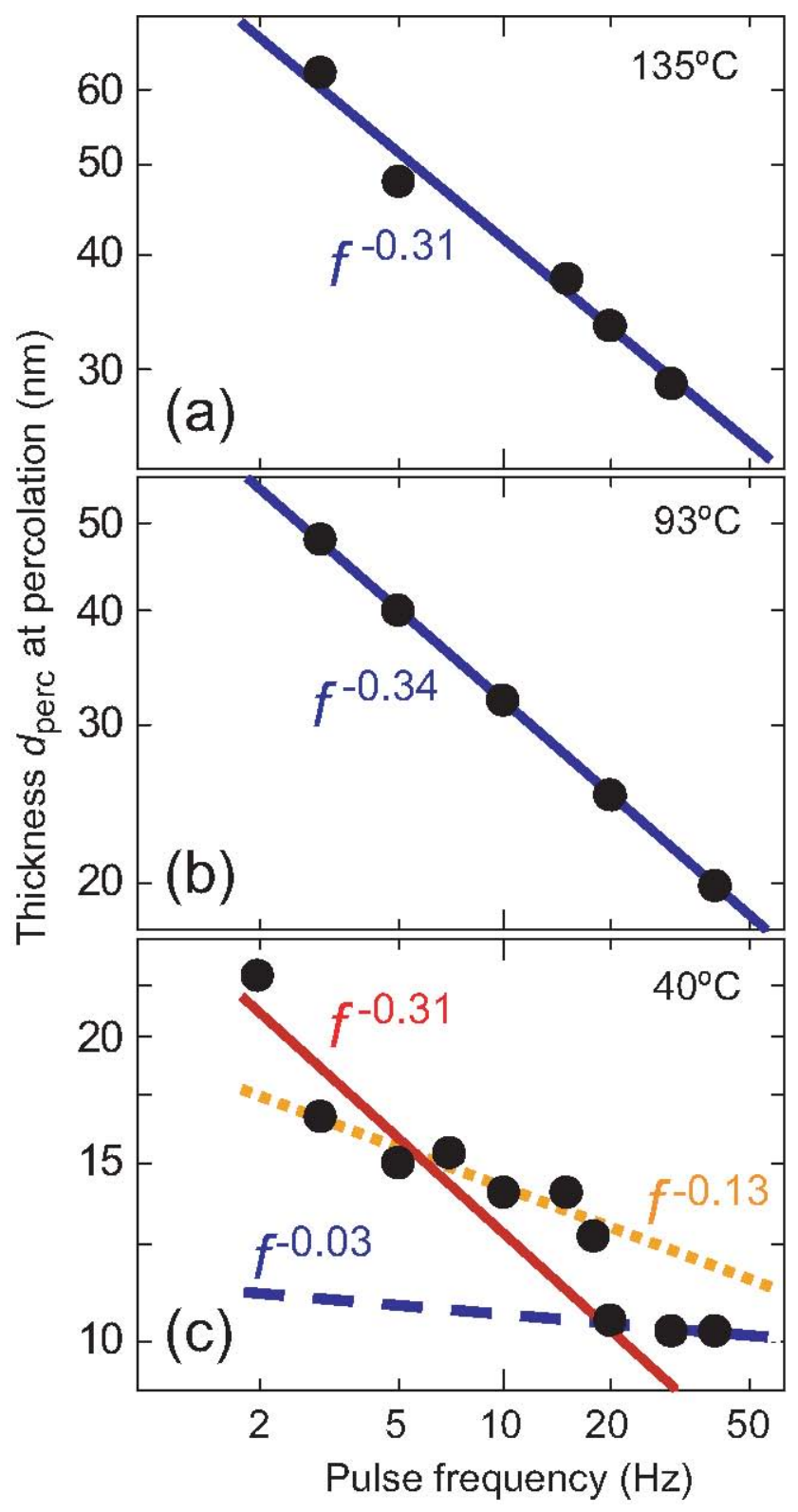

Fig. 2 


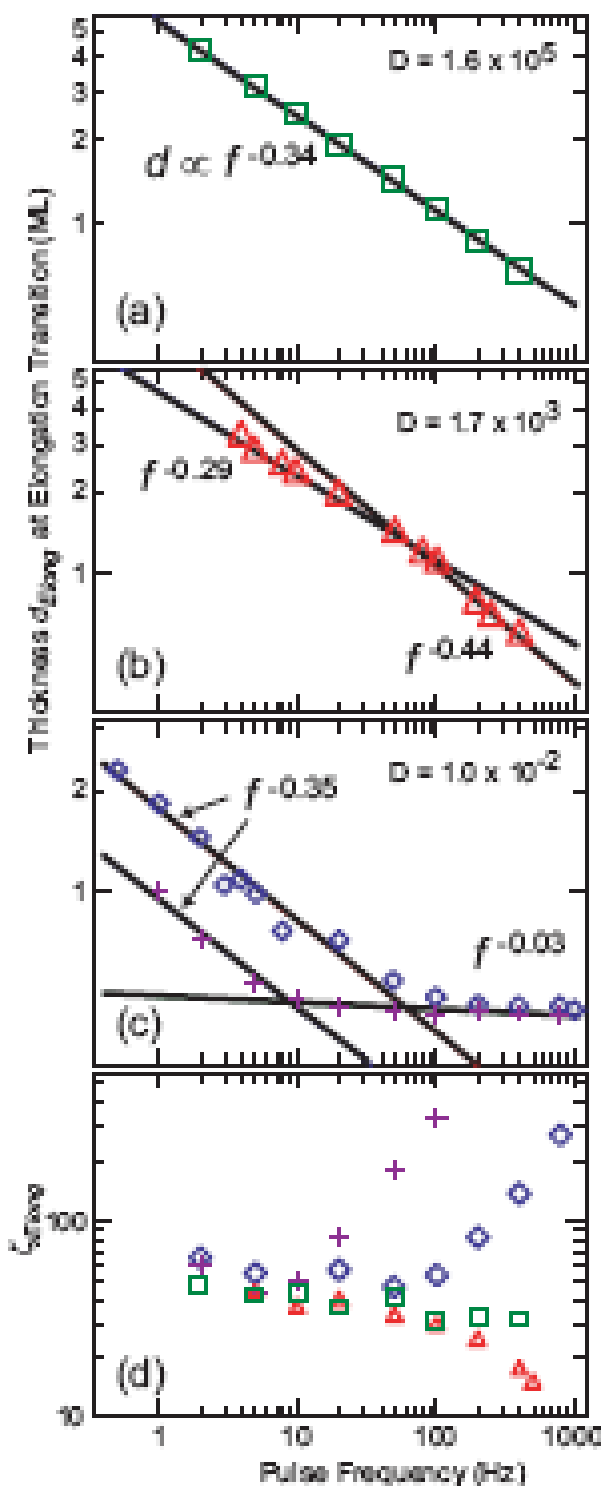

Fig. 3 

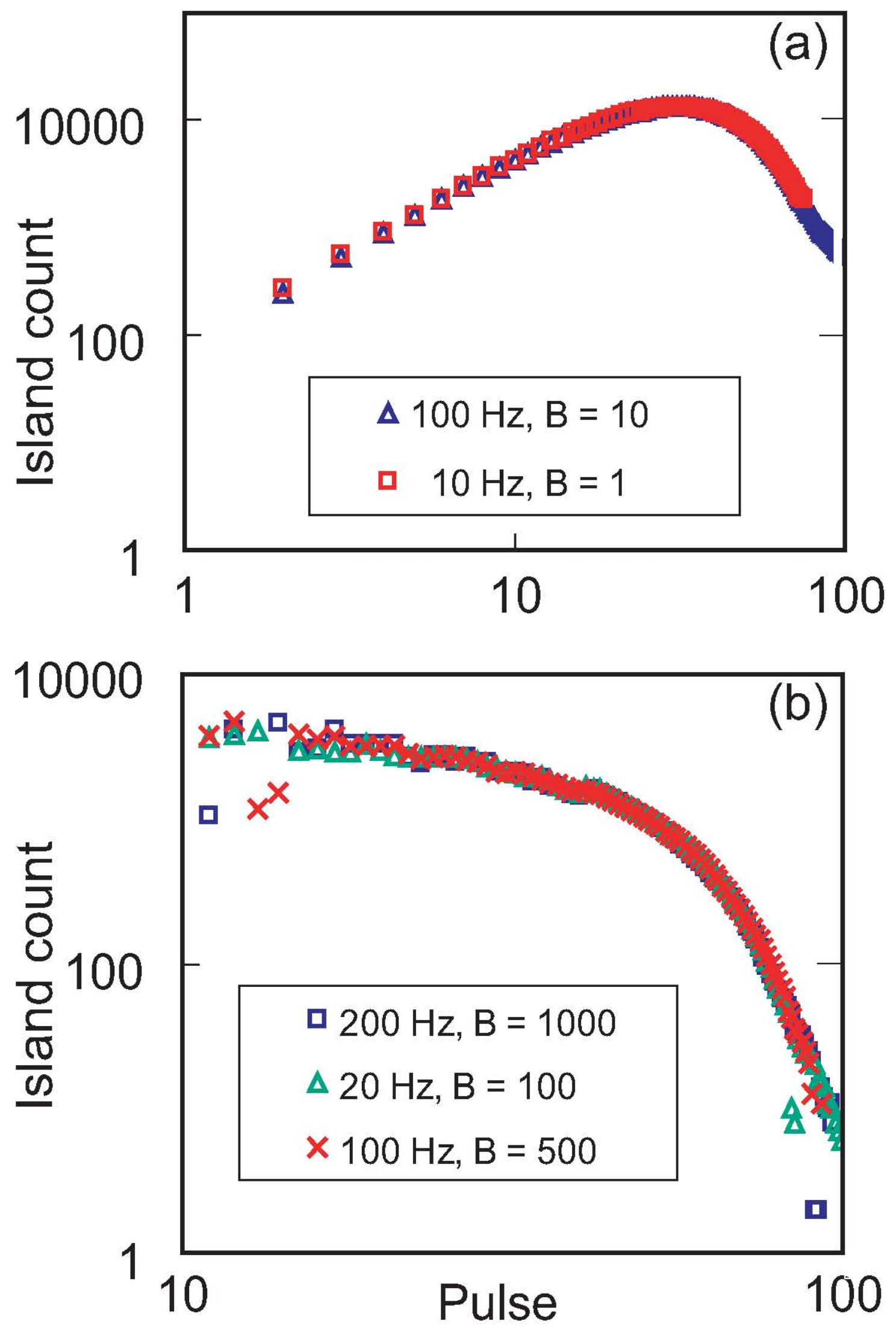

Fig. 4 